\title{
Experiencias de homofobia y adherencia al tratamiento antirretroviral (TAR) en hombres \\ que tienen sexo con hombres (HSH)
}

Experiences of homophobia and adherence to anti-retroviral treatment (ART) in men who have sex with men (MSM)

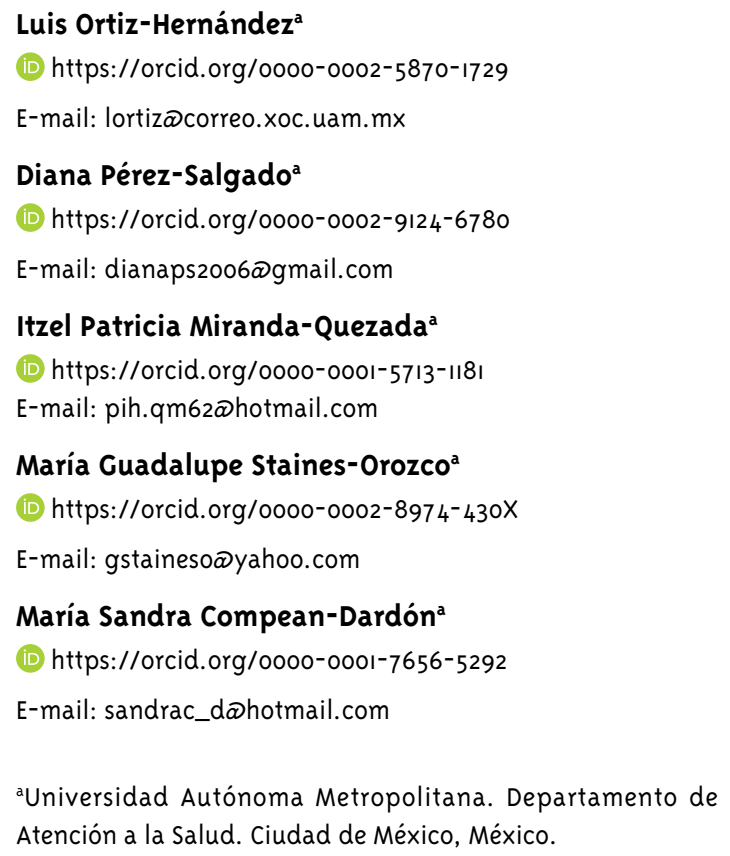
Atención a la Salud. Ciudad de México, México.

\section{Resumen}

El objetivo del estudio fue conocer la relación de las experiencias de homofobia con la adherencia al tratamiento anti-retroviral (TAR) en hombres que tienen sexo con hombres (HSH). Para ello, se realizó una encuesta transversal con una muestra por conveniencia de HSH que vivían con VIH $(n=340)$ atendidos en dos instituciones públicas de salud de la Ciudad de México. La información sobre la adherencia al TAR en el mes previo de los participantes y sus experiencias de violencia, discriminación y homofobia internalizada se recopiló mediante un cuestionario. Entre $14 \%$ y $33 \%$ de los HSH reportaron alguna experiencia de discriminación y entre $41 \%$ y $60 \%$ experimentaron violencia. Cuando los HSH habían experimentado ambos tipos de estresores, el riesgo de baja adherencia TAR fue mayor ( $\mathrm{RP}=6.49$ para mes previo) que cuando habían experimentado sólo una de ellas $(\mathrm{RP}=4.36$ para violencia y $\mathrm{RP}=5.67$ para discriminación). Los profesionales de la salud deben ser sensibles a cómo el ambiente sociocultural puede afectar las prácticas de autocuidado de HSH, incluyendo la adherencia al TAR.

Palabras clave: Cumplimiento y Adherencia al Tratamiento; VIH; Fármacos Anti-VIH; Homofobia; Minorías Sexuales y de Género.

\section{Correspondencia}


The aim of the study was to know the relationship of homophobic experiences with adherence to antiretroviral treatment (ART) in men who have sex with men (MSM) in Mexico City (CDMX). A cross-sectional study was conducted with a convenience sample of MSM who lived with HIV, treated at two public health institutions at Mexico City ( $n=340$ ). A questionnaire was applied to record information related to the adherence to ART of the participants and their experiences of violence and discrimination associated with homophobia and internalized homophobia. Between $14 \%$ and $33 \%$ of MSM reported experiences of discrimination and between $41 \%$ and $60 \%$ experienced violence. When participants had jointly experienced both types of stressors, the risk of low adherence to ART in the previous month was higher $(\mathrm{RP}=6.49)$ than when they had experienced only one of them $(\mathrm{RP}=4.36$ for violence and RP=5.67 for discrimination). Health professionals must be sensitive to how the sociocultural environment can affect selfmanagement care among MSM.

Keywords: Treatment Adherence and Compliance; HIV; Anti-HIV Agents; Homophobia; Sexual and Gender Minorities.
Hasta finales de 2019 existían alrededor de 38 millones de personas que viven con VIH (OMS, 2020). De las nuevas infecciones de VIH a nivel mundial en 2019, 60\% fueron en poblaciones clave, entre las que se incluyen a los hombres que tienen sexo con hombres (HSH). En el caso de América Latina, la infección también está concentrada en los $\mathrm{HSH}$, lo cual explica porque tienen 26 veces mayor riesgo de infectarse respecto a los heterosexuales (UNAIDS, 2020). En México, la prevalencia de VIH entre HSH es aproximadamente de $20.7 \%$ (CENSIDA, 2018), lo que implica que ellos tienen 22 veces mayor riesgo de infectarse (CENSIDA, 2019).

La adherencia al tratamiento anti-retroviral (TAR) en personas que viven con VIH les permite alcanzar la supresión viral, evitar la progresión de la enfermedad y aumentar su esperanza de vida (Nakagawa; May; Phillips, 2013). El control de la infección vía el TAR en personas que viven con VIH ha comenzado a ser considerada una forma de reducir el riesgo de transmisión (Curran; Baeten; Coates, 2012). De igual modo, se ha comenzado a recomendar que personas sin VIH tomen antirretrovirales como medida preventiva para la infección (i.e. profilaxis pre-exposición (PrEP). La PrEP está dirigida a poblaciones clave, incluyendo los HSH (Gobierno de México, 2019). Así, el estudio de la adherencia al TAR no sólo es fundamental para las personas que viven con VIH, sino también por sus posibles implicaciones para los HSH en PrEP.

Se requiere identificar los factores que pueden afectar la adherencia al TAR en HSH que viven con VIH. La adherencia ha sido definida como el grado en que el comportamiento de una persona (tal como tomar medicamentos) corresponde a las recomendaciones de un profesional de la salud (OMS, 2004). Dada su naturaleza conductual, la adherencia puede ser afectada por los procesos cognitivos y el estado de ánimo de las personas (Blashill; Perry; Safren, 2011), los cuales, a su vez, pueden ser influidos por el ambiente sociocultural (Ortiz-Hernández, 2004). Las experiencias de homofobia potencialmente pueden afectar negativamente la adherencia al TAR en $\mathrm{HSH}$, ya que se trata de las normas socioculturales que reproducen la evaluación negativa de su 
orientación sexual (Ortiz-Hernández, 2004). Los HSH experimentan diferentes formas de prejuicio (que son vividos como estresores crónicos) ya que su orientación sexual no es la dominante, además de que algunos adoptan o se piensa que adoptan rasgos femeninos (Meyer, 2003, OrtizHernández, 2004).

Para entender cómo la homofobia influye en la salud de los HSH se ha recurrido al modelo teórico de estrés de minoría, en el que se distingue entre estresores distales y proximales (Meyer, 2003). En los primeros se incluyen experiencias de violencia y discriminación; mientras que en los segundos se encuentran procesos subjetivos como homofobia internalizada. También se establecen diferencias entre la violencia estructural (normas socioculturales institucionalizadas) y la violencia interpersonal (que ocurre en la interacción entre individuos). En este modelo se considera que existen factores que pueden reducir o amortiguar el efecto de los estresores, entre los cuales se encuentran el apoyo social y la afiliación con grupos o comunidades lésbico, gay, bisexual y transgénero (LGBT).

Teóricamente, los componentes del estrés de minoría (violencia y discriminación personales y homofobia internalizada) podrían vincularse con bajo apego al TAR. La homofobia internalizada fomenta autorrechazo, miedo al rechazo social, baja autoestima, aislamiento social, conductas autodestructivas y estado de ánimo negativo (Ortiz-Hernández; García, 2005a). A su vez, los síntomas de depresión o distrés psicológico y la ideación suicida pueden estar asociadas con menor apego al TAR (Blashill; Perry; Safren, 2011).

Las experiencias de discriminación y violencia también pueden llevar a menor adherencia, ya que se asocian con distrés psicológico (Ortiz-Hernández; García, 2005b). La discriminación o la violencia pueden producir rumiación cognitiva, la cual se refiere a que las personas tienen de manera recurrente pensamientos (e.g. ¿a qué se puede atribuir la experiencia? o ¿qué tan justo o injusto es ese tipo de trato?) y emociones (casi siempre negativas) relacionadas con dichas experiencias (Hatzenbuehler; Pachankis, 2016). A su vez, la rumiación cognitiva puede condicionar que a las personas se les dificulte adoptar y mantener prácticas de autocuidado ya que mental y emocionalmente están centradas en procesar todos los aspectos relacionados con las experiencias de violencia y discriminación (Hatzenbuehler; Pachankis, 2016).

A pesar de que la epidemia de VIH está concentrada en los HSH, en general es escasa la evidencia sobre la asociación del estrés por minoría con la adherencia al TAR. Sólo identificamos dos estudios en los que se observó que la homofobia internalizada se relacionaba negativamente con la adherencia (Johnson; Carrico; Chesney, 2008, Gamarel; Neilands; Dilworth, 2015) y otro donde la discriminación por orientación sexual se asoció con ésta (Boarts; Bogart; Tabak, 2008). Sin embargo, la última relación no se presentó en otras dos muestras (Bogart; Wagner; Galvan, 2010; Galvan; Bogart; Klein, 2017). Estas investigaciones se han realizado en los Estados Unidos de Norteamérica (EUN), por lo cual se requieren estudios en la región latinoamericana.

Por todo lo anterior, el objetivo de este estudio fue conocer la relación de algunos componentes del estrés de minoría con la adherencia al TAR en HSH de la Ciudad de México. La hipótesis principal que probamos fue que los HSH que habían sufrido violencia y discriminación interpersonales o que presentaban homofobia internalizada tendrían mayor probabilidad de tener baja adherencia al TAR respecto a sus contrapartes. Una hipótesis secundaria que se planteó es que el apoyo social podría reducir el impacto de los estresores por minoría en la adherencia al TAR.

\section{Material y Métodos}

En este apartado se describen 1) el diseño del estudio y la muestra, 2) el procedimiento y las variables y 3 ) el análisis de los datos.

\section{Diseño del estudio y muestra}

Se realizó una encuesta transversal con una muestra por conveniencia de personas que viven con VIH atendidas en dos instituciones públicas de salud de la Ciudad de México. Una de las instituciones (clínica de VIH del 
Hospital Regional Lic. Adolfo López Mateos del Instituto de Seguridad y Servicios Sociales de los Trabajadores del Estado, $\mathrm{n}=100$ ) atiende a trabajadores del gobierno y sus familias; mientras que la otra (Clínica Especializada Condesa -CECde la Secretaría de Salud del Distrito Federal, $\mathrm{n}=457$ ) atiende a personas sin seguridad social que laboran en el sector informal. La CEC es el centro de referencia más importante de México ya que, de 40,ooo personas que se estimaba vivían con VIH en la Ciudad de México, el 23\% (n=9,114) eran atendidas ahí (México, 2013). El estudio se llevó a cabo en el periodo comprendido de octubre de 2011 a noviembre de 2012.

Del total de participantes en el estudio $(n=557)$, 340 eran HSH. En este reporte solo se presentan los datos de éstos últimos. Las características de toda la muestra fueron publicadas previamente (Ortiz-Hernández; Pérez-Salgado; CompeanDardón, 2016). Con la siguiente pregunta se identificó a los HSH: “¿Con quién ha tenido relaciones sexuales?”. Existían siete opciones de repuesta (e.g. "no ha tenido relaciones sexuales", "sólo con mujeres", "hombres y mujeres por igual" o "sólo con hombres"). Se excluyeron a los que seleccionaron las dos primeras opciones.

El proyecto fue aprobado por el Comité de Ética en Investigación de la Universidad Autónoma Metropolitana, unidad Xochimilco.
Se solicitó el consentimiento informado por escrito de cada participante.

\section{Procedimiento y variables}

Se aplicó un cuestionario para registrar información sobre la adherencia al TAR de los participantes y sus experiencias de violencia, discriminación (estresores distales) y homofobia internalizada (estresor proximal). El cuestionario fue aplicado por enfermeras capacitadas en técnicas de entrevista.

La escala de homofobia internalizada consistía en 9 ítems que se presentan en la Tabla 1. Esta escala desarrollada en Estados Unidos de Norteamérica (Ortiz-Hernández, 2005) ha sido traducida y adaptada para su uso en México (Ortiz-Hernández, 2005, OrtizHernández; García, 2005a). Existen cinco opciones de respuestas, ie. de "totalmente en desacuerdo" a "totalmente de acuerdo", a las cuales se les asignó una puntuación de uno a cinco. Con las respuestas de los participantes, se realizó un análisis factorial exploratorio (ver última columna de la Tabla 1). Solo emergió un factor y todos los ítems tuvieron una carga factorial de >0.40. El alfa de Cronbach fue de 0.94 . Se sumaron los puntajes de las respuestas y se creó una variable de tres categorías: sin homofobia (9 puntos), homofobia internalizada leve (10 a 18 puntos) y homofobia internalizada (19 a 39 puntos).

Tabla I - Distribución de HSH que vivían con VIH de la Ciudad de México de acuerdo con una escala de homofobia internalizada

\begin{tabular}{|c|c|c|c|c|c|c|}
\hline & TDA & DA & A-DA & A & TA & nálisis \\
\hline & $\%$ & $\%$ & $\%$ & $\%$ & $\%$ & factorial \\
\hline Eigen-value & & & & & & 6.42 \\
\hline$\%$ Varianza & & & & & & 71.3 \\
\hline $\begin{array}{l}\text { Con frecuencia siente que es mejor evitar involucrarse personal y } \\
\text { socialmente con otros homo y bisexuales }\end{array}$ & 59.4 & 27.4 & 6.5 & 4.1 & 2.6 & 0.50 \\
\hline Ha tratado que ya no le atraigan los hombres & 64.7 & 29.4 & 2.6 & 2.4 & 0.9 & 0.83 \\
\hline $\begin{array}{l}\text { Si alguien le ofreciera la oportunidad de ser completamente } \\
\text { heterosexual, usted aceptaría }\end{array}$ & 65.3 & 27.9 & 2.9 & 2.4 & 1.5 & 0.83 \\
\hline Usted desearía ya no ser homosexual o bisexual & 64.7 & 27.9 & 4.4 & 2.1 & 0.9 & 0.90 \\
\hline Se siente extraño consigo mismo debido a que es homosexual o bisexual & 62.6 & 30.0 & 5.6 & 1.2 & 0.6 & 0.90 \\
\hline
\end{tabular}


Tabla I - Continuación

\begin{tabular}{|c|c|c|c|c|c|c|}
\hline & TDA & DA & A-DA & A & TA & Análisis \\
\hline & $\%$ & $\%$ & $\%$ & $\%$ & $\%$ & factorial \\
\hline Desearía poder desarrollar más sentimientos eróticos por las mujeres & 61.8 & 27.9 & 6.8 & 1.8 & 1.8 & 0.92 \\
\hline Siente que ser homosexual o bisexual es un defecto personal & 63.2 & 30.3 & 5.6 & 0.6 & 0.3 & 0.87 \\
\hline Le gustaría tener ayuda profesional para convertirse en heterosexual & 62.9 & 30.6 & 4.4 & 1.5 & 0.6 & 0.88 \\
\hline Ha tratado de sentirse más atraído sexualmente por las mujeres & 62.1 & 29.7 & 5.3 & 1.8 & 1.2 & 0.87 \\
\hline
\end{tabular}

Abreviaturas: HSH, hombres que tienen sexo con hombres; TDA, totalmente en desacuerdo; DA, en desacuerdo; A-DA, tan de acuerdo como en desacuerdo; A, de acuerdo; y TA, Totalmente de acuerdo.

Para evaluar las experiencias de violencia y discriminación interpersonales se utilizó una escala de diez preguntas cuyo contenido se presenta en la Tabla 2. Esta escala derivó de un estudio previo con población LGBT de la Ciudad de México (OrtizHernández; García, 2005a). A los participantes se preguntó si alguna vez en la vida les habían sucedido las experiencias de violencia o discriminación debido "a que otras personas han creído, sospechado o sabido que usted podría ser o es bisexual u homosexual”. Existían cuatro opciones de respuesta: nunca, una vez, dos o tres veces y cuatro veces o más, a las que se asignó una puntuación de uno a cuatro. Las respuestas de los participantes fueron sometidas a análisis factorial en el que emergieron dos factores (ver últimas columnas de la Tabla 2). Considerando que las experiencias de violencia y discriminación pueden estar vinculadas se optó por rotación oblicua promax. El primer factor incluyó del ítem 6 al 10 (con cargas factoriales $>0.40$ en este factor), fue nombrado "violencia interpersonal" y tuvo un alfa de Cronbach de 0.90 . El segundo factor contuvo a los ítems 1 a 5, se denominó "discriminación interpersonal" y tuvo un alfa de Cronbach de o.81. Se sumaron las respuestas de los ítems correspondientes a cada factor. Se sumó la puntuación de las respuestas a las preguntas que quedaron en cada factor y finalmente se crearon dos variables dicotómicas utilizando como punto de corte la mediana de cada factor. Por arriba de la mediana ( 5 para discriminación y 6 para violencia) se consideró que habían experimentado violencia o discriminación.

\section{Tabla 2 - Distribución de HSH que vivían con VIH de la Ciudad de México de acuerdo con la escala de violencia} y discriminación

\begin{tabular}{|c|c|c|c|c|c|c|}
\hline & $\mathrm{N}$ & 1 & $2-3$ & ${ }^{3} 4$ & $\mathrm{Fl}_{1}$ & $\mathrm{~F}_{2}$ \\
\hline & $\%$ & $\%$ & $\%$ & $\%$ & & \\
\hline Eigen value & & & & & 4.98 & 1.74 \\
\hline$\%$ Varianza & & & & & 49.8 & 17.4 \\
\hline $\begin{array}{l}\text { I. Le negaron un ascenso de puesto de trabajo, o aumento de salario, lo } \\
\text { despidieron, le negaron un trabajo o le ha sucedido una situación similar } \\
\text { 2. Le negaron el ingreso a una escuela, en la escuela le han reducido una }\end{array}$ & $74 \cdot 4$ & 17.6 & 5.9 & 2.1 & -0.06 & 0.86 \\
\hline $\begin{array}{l}\text { calificación, no le han reconocido su desempeño, le han maltratado o le ha } \\
\text { sucedido una situación similar }\end{array}$ & 86.2 & 7.9 & 5.6 & 0.3 & -0.07 & 0.86 \\
\hline $\begin{array}{l}\text { 3. Le han negado los servicios de salud en consultorios, clínicas u hospitales, } \\
\text { ha sufrido regaños, humillación o maltrato por parte de algún médico, } \\
\text { enfermeras u otros profesionales de salud }\end{array}$ & 83.8 & 10.0 & 5.0 & 1.2 & -0.09 & 0.82 \\
\hline $\begin{array}{l}\text { 4. Le han negado los servicios en restaurantes, cines u otros servicios, ha } \\
\text { sufrido regaños, humillación o maltrato por parte de meseros, vendedores, } \\
\text { empresarios, etc. }\end{array}$ & 80.0 & 9.7 & 7.9 & 2.4 & 0.40 & 0.49 \\
\hline 5. La policía lo ha detenido, expulsado de algún lugar, amenazado, o extorsionado & 66.8 & 17.1 & 12.9 & 3.2 & 0.39 & 0.49 \\
\hline
\end{tabular}




\begin{tabular}{|c|c|c|c|c|c|c|}
\hline & $\mathrm{N}$ & 1 & $2-3$ & ${ }^{3} 4$ & $\mathrm{Fl}$ & $\mathrm{F}_{2}$ \\
\hline & $\%$ & $\%$ & $\%$ & $\%$ & & \\
\hline $\begin{array}{l}\text { 6. Le insultaron o agredieron verbalmente con palabras como "puto", } \\
\text { "maricón", etc. }\end{array}$ & 39.4 & 19.7 & 22.6 & 18.2 & 0.71 & 0.06 \\
\hline 7. Le amenazaron con violencia & 54.7 & 15.9 & 18.2 & 11.2 & 0.91 & 0.01 \\
\hline 8. Le golpearon, pegaron o agredieron físicamente & 58.2 & 15.9 & 15.6 & 10.3 & 0.89 & 0.01 \\
\hline 9. Le acosaron sexualmente usando palabras obscenas o sexuales & 46.5 & 18.8 & 18.8 & 15.9 & 0.90 & -0.06 \\
\hline 10. Le robaron, asaltaron o le dañaron o maltrataron un objeto suyo & 47.6 & 19.7 & 15.6 & 17.1 & 0.85 & -0.16 \\
\hline
\end{tabular}

Abreviaturas: HSH, hombres que tienen sexo con hombres; N, nunca; I, una vez; 2-3, dos o tres veces; ${ }^{3} 4$, cuatro o más veces, $\mathrm{Fl}$, factor I denominado violencia; $\mathrm{F} 2$, factor 2, nombrado discriminación.

La adherencia al TAR fue evaluada con una pregunta que indagaba si la persona había omitido la toma de medicamentos antirretrovirales durante el mes previo a la entrevista. Este tiempo de referencia fue elegido para reducir el sesgo de memoria. Las opciones de respuesta eran sí o no. Cuando la respuesta fue afirmativa, se preguntó cuántas veces había omitido tomar el medicamento. La redacción de estas preguntas fue tomada de estudios previos (OrtizHernández; Pérez-Salgado; Compean-Dardón, 2016). No se indagó el número de pastillas ya que los esquemas de TAR eran más simples y, por tanto, preguntarlo no agregaba más información. Al momento del estudio el TAR recomendado consistía en dos inhibidores de la transcriptasa reversa análogos a nucleósidos (que con frecuencia implican una pastilla) y un inhibidor de la transcriptasa reversa no nucleósido (una pastilla) (CENSIDA, 2012). A partir del número de veces que se había omitido tomar el TAR, se creó una nueva variable que identifica la baja adherencia durante ese periodo. Se denominó baja adherencia cuando la persona había omitido más del $5 \%$ de las dosis prescritas de medicamentos. Con una adherencia 95\% se alcanza el control de la infección (Nakagawa; May; Phillips, 2013).

La evaluación del apoyo social se realizó mediante las preguntas con escala Likert del cuestionario MOS (Medical Outcomes Study), el cual fue desarrollado en Estados Unidos (Sherbourne; Stewart, 1991) y posteriormente fue traducido al español y validado su uso en personas que viven con VIH (Remor, 2003). La escala consiste en 19 preguntas tipo Likert y a las opciones de respuesta se les asignó una puntuación de 1 (nunca) a 5 (siempre). En un análisis factorial de las respuestas de los HSH se observó un factor que incluía a las preguntas 2, 4 a 10, 12, 15, 16, 18 a 20. El Alpha de
Cronbach fue de 0.97. Las respuestas de los ítems se sumaron y la variable resultante se dicotomizó usando la mediada (62) como punto de corte.

Como covariables se consideraron la edad, el estado civil y la orientación sexual de los participantes, la escolaridad del jefe de familia y la inseguridad alimentaria. Se consideró la identidad que han asumido los participantes, para lo cual se aplicó la siguiente pregunta: “¿Usted piensa qué es...?” Las opciones de respuesta eran: "heterosexual solamente", "bisexual, pero más heterosexual", "bisexual", "bisexual pero más homosexual", "homosexual solamente" y "no está seguro". Los que respondieron "homosexual solamente" fueron considerados como "homosexuales" y al resto como "bisexuales". Las cinco opciones para estado civil incluidas en el cuestionario fueron clasificadas en tres grupos: solteros; casado o en unión libre; y divorciado y viudo.

La inseguridad alimentaria y la escolaridad del jefe de familia (i.e. la persona que sostenía económicamente a la familia) fueron utilizadas como indicadores de la posición socioeconómica. Existían ocho opciones para la escolaridad que fueron agrupados en tres categorías: secundaria o menos (que corresponden a 9 años o menos de educación), bachillerato (12 años de educación) y licenciatura o posgrado (15 o más años de educación). La forma en que se evaluó la inseguridad alimentaria ha sido descrita previamente (Ortiz-Hernández; Pérez-Salgado; Compean-Dardón, 2016). La posición socioeconómica puede ser un confusor ya que peores condiciones socioeconómicas se relacionan tanto con niveles más altos de homofobia internalizada (McGarrity, 2014) como de baja adherencia al TAR (Ortiz-Hernández; Pérez-Salgado; Compean-Dardón, 
2016). Por tanto, la relación entre estas dos últimas podría deberse por su correlación con la primera.

Como covariables relacionadas con el TAR se consideró si los participantes habían cambiado de TAR y el tiempo que lo han consumido. Estas variables son relevantes ya que las personas con más tiempo bajo TAR utilizaron esquemas con más pastillas, además de que pueden experimentar cansancio por el autocuidado.

\section{Análisis de datos}

Para el análisis descriptivo, estimamos la prevalencia de baja adherencia. Para determinar si la adherencia al TAR estaba asociada con alguno de los estresores distales y proximales, realizamos un análisis bivariado usando la prueba de chi cuadrada para conocer si existían diferencias estadísticamente significativas entre grupos definidos de acuerdo con la homofobia internalizada y las experiencias de discriminación y violencia. Un valor de $p<0.050$ se consideró como estadísticamente significativo.

Se estimaron modelos de regresión Poisson usando como exposiciones a las experiencias de discriminación y violencia y como evento a la baja adherencia al TAR. Dado que la violencia y la discriminación se asociaron con las variables de adherencia de manera separada, estimamos modelos donde se incorporó su interacción. A partir de los modelos se estimaron razones de prevalencia (RP). Para probar la hipótesis secundaria se estimaron modelos donde se incluyeron la interacción del apoyo social con la violencia y con la discriminación. Para facilitar la interpretación de estas interacciones, se graficaron las probabilidades ajustadas de baja adherencia estimadas a partir de los modelos de regresión. Todos los modelos fueron ajustados por características sociodemográficas y covariables relacionadas con el TAR ya que podrían actuar como confusores. El análisis estadístico se realizó con el programa STATA versión 15.

\section{Resultados}

La media de edad fue 34.5 años (desviación estándar 10.2); la mayoría eran solteros, seguidos por los que estaban casados o en unión libre (Tabla 3). La escolaridad del jefe de familia más común fue bachillerato, seguido por secundaria.
Más de la mitad presentó algún grado de inseguridad alimentaria. Tres cuartas partes de los participantes se identificaban como homosexuales.

\begin{tabular}{|c|c|}
\hline Estado civil & $\%$ \\
\hline Soltero & 79.4 \\
\hline Casado o unión libre & $15 \cdot 3$ \\
\hline Divorciado o viudo & 5.3 \\
\hline \multicolumn{2}{|l|}{ Orientación sexual } \\
\hline Homosexual & $75 \cdot 3$ \\
\hline Bisexual & 24.7 \\
\hline \multicolumn{2}{|l|}{ Apoyo social } \\
\hline \multicolumn{2}{|l|}{ Escolaridad del jefe de familia } \\
\hline "Secundaria & 33.8 \\
\hline Preparatoria o bachillerato & 40.0 \\
\hline Licenciatura o posgrado & 26.2 \\
\hline \multicolumn{2}{|l|}{ Inseguridad alimentaria } \\
\hline Seguridad alimentaria & 40.3 \\
\hline Inseguridad alimentaria & 59.7 \\
\hline
\end{tabular}

$\mathrm{HSH}$, hombres que tienen sexo con otros hombres.

Entre el $1 \%$ y $6 \%$ de lo HSH reportaron alguna experiencia de homofobia internalizada, mientras que entre el $14 \%$ y el $33 \%$ reportaron alguna experiencia de discriminación por homofobia y entre el $41 \%$ y el $60 \%$ experimentaron alguna forma de violencia por homofobia (Tablas 1 y 2 ).

La prevalencia de baja adherencia no difirió de acuerdo a la homofobia internalizada (Tabla 4 ). Las personas que habían sufrido discriminación o violencia presentaron mayores frecuencias de baja adherencia, comparadas con las que no habían tenido dichas experiencias. Los HSH que habían sufrido alguna experiencia de discriminación o violencia tuvieron más tiempo en TAR.

Después de ajustar por características sociodemográficas y covariables relacionadas con el TAR, se observó que los participantes que habían sufrido violencia o discriminación tuvieron mayor probabilidad de baja adherencia al TAR respecto a los que no tuvieron estas experiencias (Tabla 5). El riesgo de baja adherencia al TAR fue mayor cuando los participantes habían experimentado tanto discriminación como violencia respecto a cuando habían experimentado solo una de ellas. 
Tabla 4 - Prevalencia de baja adherencia al tratamiento antirretroviral de acuerdo con estresores distales y un estresor proximal en HSH que vivían con VIH de la ciudad de México

\begin{tabular}{|c|c|c|c|c|c|c|c|c|c|c|c|}
\hline \multirow{4}{*}{$(n)$} & \multirow[b]{3}{*}{$\begin{array}{l}\text { Total } \\
(340)\end{array}$} & \multicolumn{4}{|c|}{ Estresor proximal } & \multicolumn{6}{|c|}{ Estresores distales } \\
\hline & & \multicolumn{4}{|c|}{ Homofobia internalizada } & \multicolumn{3}{|c|}{$\begin{array}{c}\text { Violencia } \\
\text { interpersonal }\end{array}$} & \multicolumn{3}{|c|}{$\begin{array}{l}\text { Discriminación } \\
\text { interpersonal }\end{array}$} \\
\hline & & $\begin{array}{c}\text { SHI } \\
(168)\end{array}$ & $\begin{array}{c}\text { HIL } \\
\text { (II8) }\end{array}$ & $\begin{array}{c}\mathrm{HI} \\
(45)\end{array}$ & & $\begin{array}{c}\text { No } \\
(183)\end{array}$ & $\begin{array}{c}\text { Sí } \\
(157)\end{array}$ & & $\begin{array}{c}\text { No } \\
(183)\end{array}$ & $\begin{array}{c}\text { Sí } \\
(157)\end{array}$ & \\
\hline & $\%$ & $\%$ & $\%$ & $\%$ & $p$ & $\%$ & $\%$ & $p$ & $\%$ & $\%$ & $p$ \\
\hline Baja adherencia & 5.9 & 6.0 & 4.1 & 10.2 & 0.303 & $3 \cdot 3$ & 8.9 & 0.028 & 2.7 & 9.6 & 0.008 \\
\hline \multirow[t]{2}{*}{ Ha cambiado de tratamiento antirretroviral } & 41.1 & 45.8 & 43.1 & 20.4 & 0.005 & 39.9 & 42.7 & 0.603 & 39.3 & $43 \cdot 3$ & 0.459 \\
\hline & M & M & M & M & & M & M & & M & M & \\
\hline Tiempo en tratamiento, años & 5.12 & 5.65 & 4.59 & 4.65 & 0.146 & 4.46 & 5.89 & 0.007 & 4.61 & 5.72 & 0.036 \\
\hline
\end{tabular}

Abreviaturas: HSH, hombres que tienen sexo con otros hombres; SHI, sin homofobia; HIL, homofobia internalizada leve; HI, homofobia internalizada; M, media.

La interacción del apoyo social con la violencia no fue significativa. La interacción del apoyo social con la discriminación sí fue significativa (Tabla 5). Entre los HSH que contaban con apoyo social, la probabilidad de baja adherencia fue 1.63 veces más alta entre los que sufrieron discriminación (probabilidad: o.o31) respecto a los que no la sufrieron (probabilidad: o.019, ver Gráfico 1). En cambio, entre los que no contaban con apoyo social la probabilidad de baja adherencia fue de 3.05 veces más alta entre los que sufrieron discriminación (probabilidad: 0.156) respecto a los que no (probabilidad: 0.051).

Tabla 5 - Modelos de regresión en los que las exposiciones fueron los estresores distales y el evento fue la baja adherencia al tratamiento antirretroviral en HSH que vivían con VIH de la ciudad de México

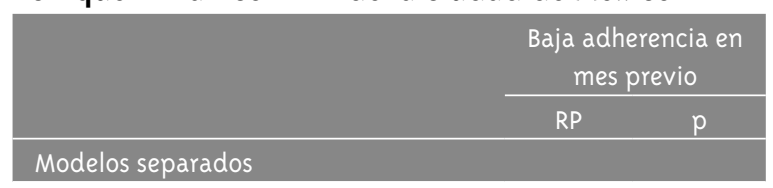

Violencia

\begin{tabular}{lcc} 
No & Ref. & Ref. \\
Sí & 2.60 & 0.062 \\
$\begin{array}{l}\text { Discriminación } \\
\text { No }\end{array}$ & Ref. & Ref. \\
Sí & 3.25 & 0.025 \\
\hline Modelo de interacción I & & \\
Sin discriminación ni violencia & Ref. & \\
Solo violencia & 4.36 & 0.115 \\
Solo discriminación & 5.67 & 0.050 \\
Discriminación y violencia & 6.49 & 0.018 \\
\hline
\end{tabular}

\section{Tabla 5-Continuación}

\begin{tabular}{llc} 
& \multicolumn{2}{c}{$\begin{array}{c}\text { Baja adherencia en } \\
\text { mes previo }\end{array}$} \\
\cline { 2 - 3 } & RP & $p$ \\
\hline Modelo de interacción 2 & & \\
No apoyo social y no violencia & Ref. \\
No apoyo social y sí violencia & 2.67 & 0.161 \\
Sí apoyo social y no violencia & 0.45 & 0.348 \\
Sí apoyo social y sí violencia & 1.11 & 0.885 \\
Modelo de interacción 3 & & \\
No apoyo social y no discriminación & Ref. \\
No apoyo social y sí discriminación & 8.12 & 0.048 \\
Sí apoyo social y no discriminación & 1.63 & 0.667 \\
Sí apoyo social y sí discriminación & 2.66 & 0.379 \\
\hline
\end{tabular}

Abreviaturas: HSH, hombres que tienen sexo con otros hombres; RP, razón de prevalencias; Ref., grupo de referencia. Todos los modelos están ajustados por edad, estado civil y orientación sexual de los participantes, escolaridad del jefe de familia e inseguridad alimentaria, tiempo en tratamiento y si han cambiado tratamiento.

Gráfico I - Probabilidad de baja adherencia al tratamiento antirretroviral de acuerdo con apoyo social y discriminación en HSH que vivían con VIH de la ciudad de México

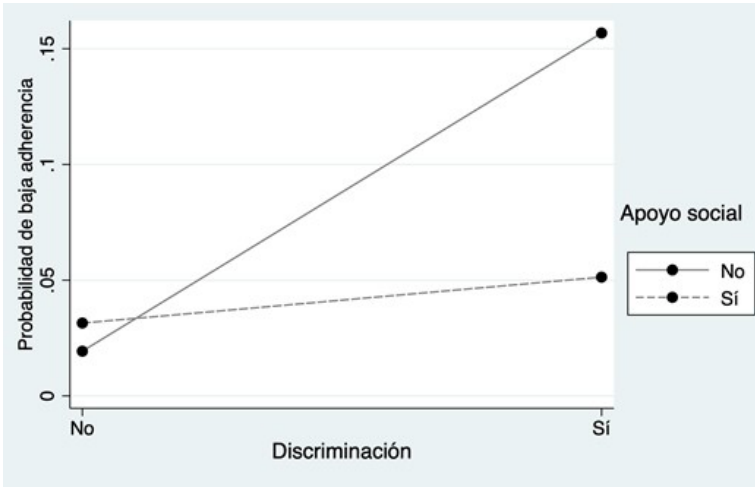




\section{Discusión}

Los resultados de la investigación apoyan parcialmente nuestra hipótesis principal ya que los HSH que habían sufrido violencia y discriminación tuvieron mayor probabilidad de tener baja adherencia al TAR. De igual modo, obtuvimos apoyo empírico para sugerir que el apoyo social puede tener un efecto amortiguador ya que entre los HSH que contaban con él fue menos fuerte la relación de las experiencias de discriminación con la adherencia al TAR. Sin embargo, en los HSH que participaron en el estudio, la homofobia internalizada no se asoció con la adherencia al TAR.

En los HSH que viven con VIH de la Ciudad de México no existió asociación entre la homofobia internalizada y la adherencia al TAR. La escasa evidencia sobre la relación de la homofobia internalizada con la adherencia al TAR en HSH ha sido inconsistente ya que en un estudio no se observó la relación (Gamarel; Neilands; Dilworth, 2015); pero en otro sí (Johnson; Carrico; Chesney, 2008). Una de las posibles razones por las que no se encontró asociación en nuestro estudio es que evaluamos una forma extrema de homofobia internalizada, la cual cada vez es menos frecuente (tal como se observó en los HSH de la ciudad de México). La homofobia internalizada puede manifestarse de diferentes formas, una de las más extremas es el querer modificar la orientación sexual por considerarla una cualidad totalmente inaceptable; al mismo tiempo, también existen expresiones más sutiles como el experimentar vergüenza por ser un varón homosexual o tener actitudes negativas a la feminidad en los varones, sin que ello implique el deseo de modificar la orientación sexual (Ortiz-Hernández; García, 2005a). Las expresiones de estas manifestaciones se han modificado dados los cambios culturales recientes. Existe un ambiente de mayor aceptación hacia la homosexualidad debido a los cambios institucionales y legales que han ocurrido en los últimos años (ej. se incluyó en el código civil el matrimonio entre personas del mismo sexo) (Mendoza-Pérez; Ortiz-Hernández, 2020). Este ambiente menos adverso puede propiciar que cada vez sea menos frecuente que los HSH vivan su orientación sexual en términos tan negativos que deseen cambiarla.
Sin embargo, la estigmatización de la homosexualidad persiste en formas sutiles (Mendoza-Pérez; Ortiz-Hernández, 2020). Por ello, es probable que ahora la homofobia internalizada también se exprese en formas sutiles. Por ejemplo, ahora la homofobia internalizada en HSH podría asociarse con experiencias de incomodidad, vergüenza o culpa por su orientación sexual (Ortiz-Hernández, 2005). También se ha sugerido que la homofobia internalizada puede manifestarse como actitud negativa a los varones femeninos (Mendoza-Pérez; Ortiz-Hernández, 2020). Futuras investigaciones podrían evaluar estas manifestaciones de homofobia internalizada y verificar si están asociadas con la adherencia al TAR.

Nuestros resultados sugieren que la discriminación y la violencia por la orientación sexual siguen siendo experiencias comunes entre HSH. Las formas más comunes de discriminación fueron maltrato por policías y trato diferencial en el trabajo; mientras que las agresiones verbales y el acoso sexual fueron las violencias más comunes. En sociedades occidentales sistemáticamente se ha observado que la población LGBT es objeto de violencia debido a su orientación sexual o identidad de género (Mendoza-Pérez; Ortiz-Hernández, 2020).

En HSH que viven con VIH de Los Ángeles, se observó una tendencia a tener menos adherencia al TAR entre aquellos que habían experimentado discriminación sexual, aunque las diferencias no fueron significativas (Bogart; Wagner; Galvan, 2010). Entre los HSH de la Ciudad de México las experiencias de discriminación y violencia por homofobia se asociaron con mayor probabilidad de no adherencia al TAR. Estas variables pueden tener un efecto acumulativo, es decir, el riesgo de baja adherencia es mayor cuando se han tenido ambas experiencias respecto a cuando se presenta solo una. Al sufrir violencia y discriminación por homofobia, los HSH pueden percibir que la mayoría de las personas tienen actitudes negativas hacia su orientación sexual (Mendoza-Pérez; Ortiz-Hernández, 2020). Esto podría propiciar que los HSH comiencen a ser conscientes de que en el futuro pueden ser víctimas de violencia y discriminación. Estas expectativas pueden generar que vigilen sus conductas buscando reducir esa posibilidad, lo cual 
puede ser acompañado de ansiedad (García; Parker; Parker, 2016). La expectativa de rechazo también es un estresor crónico (Meyer, 2003). La discriminación en los servicios de salud especialmente podría tener un efecto negativo en la adherencia pues la falta de confianza en los médicos se relaciona con menor adherencia al TAR (Compeán; Pérez-Salgado, 2015). Estas situaciones son emocionalmente desgastantes y cognitivamente absorbentes, por lo cual los HSH pueden tener menor capacidad para mantener prácticas de autocuidado (Hatzenbuehler; Pachankis, 2016).

Como esperábamos, la presencia de apoyo social amortiguó el efecto de la discriminación sobre la adherencia al TAR. El apoyo social puede reducir el efecto de la discriminación ya que las personas pueden tener la posibilidad de manejar las emociones negativas que esas experiencias generan, así como alternativas para afrontar el trato injusto (Doan Van; Mereish; Woulfe, 2019). Futuras investigaciones podrían explorar otros factores protectores como la resiliencia o su participación política. La primera implica que el HSH tiene recursos personales para afrontar las experiencias de prejuicio; mientras que la segunda no sólo requiere la aceptación personal de la orientación sexual, sino también un cuestionamiento colectivo de la homofobia cultural, con lo cual se rechazan las evaluaciones negativas de la homosexualidad. Estudiar estos factores implica reconocer que los HSH también son agentes activos que dan respuesta a los procesos que afectan su salud y bienestar.

La representatividad de la muestra es una limitación de nuestro estudio, pues sólo incluimos dos instituciones públicas de la Ciudad de México. Al mismo tiempo, una de las instituciones donde se realizó el estudio atiende alrededor de una cuarta parte de las personas que viven con VIH de la Ciudad de México. Una limitación adicional en nuestro estudio es que usamos el reporte de los participantes mediante preguntas que no han sido validadas para su uso en personas que viven con VIH. Sin embargo, se ha observado (Nieuwkerk; Oort, 2005) que existe correlación entre el reporte de adherencia y los niveles de carga viral, lo cual refleja que la información que proporcionan las personas que viven con VIH es adecuada.
En síntesis, observamos que las experiencias de discriminación y violencia por homofobia se asociaron con menor adherencia al TAR en HSH de la Ciudad de México. La presencia de apoyo social amortiguó el efecto de la discriminación sobre la adherencia al TAR. No existió asociación entre la homofobia internalizada y la adherencia al TAR. Dados los cambios institucionales y legales recientes, que propician un ambiente de mayor aceptación hacia la homosexualidad, se hace necesario evaluar formas sutiles de homofobia internalizada en la población estudiada.

\section{Consideraciones finales}

Nuestros resultados sugieren que los profesionales de la salud deben ser sensibles a cómo el ambiente sociocultural puede afectar negativamente las prácticas de autocuidado. Convendría, entonces, que los clínicos exploren con los HSH que viven con VIH si han experimentado discriminación o violencia ya que esto puede limitar la adherencia al TAR. También puede evaluarse y promoverse la existencia de redes de apoyo en torno a los HSH ya que éstas pueden amortiguar el efecto de la discriminación sobre la adherencia al TAR. A nivel poblacional, se requieren medidas que contribuyan a des-estigmatizar la homosexualidad, ya que los prejuicios contra esta orientación sexual son los que originan los actos de violencia y discriminación (Mendoza-Pérez; Ortiz-Hernández, 2020). Los cambios legales e institucionales que han ocurrido para proteger los derechos de la población LGBT en México seguirán contribuyendo a que la población resignifique las orientaciones sexuales e identidades de género no normativas. Aunado a lo anterior, se requieren campañas de medios en los que se muestre la diversidad sexual y genérica como parte de la naturaleza humana. También se pueden emitir mensajes sobre las consecuencias que tiene en las personas la violencia y discriminación por homofobia.

\section{Referencias}

BLASHILL, A. J.; PERRY, N.; SAFREN, S. A.

Mental health: a focus on stress, coping, y mental illness as it relates to treatment retention, 
adherence, y other health outcomes. Current HIV/ AIDS Reports, Berlin, v. 8, n. 4, p. 215-222, 2011. DOI: 10.1007/s11904-011-0089-1

BOARTS, J.; BOGART, L.; TABAK, M. et al. Relationship of race-, sexual orientation-, y HIVrelated discrimination with adherence to HIV treatment: a pilot study. Journal of Behavioral Medicine, Berlin, v. 31, n. 5, p. 445-451, 2008. DOI: 10.1007/s10865-008-9169-o

BOGART, L.; WAGNER, G.; GALVAN, F. et al. Longitudinal relationships between antiretroviral treatment adherence y discrimination due to HIV-serostatus, race, y sexual orientation among African-American men with HIV. Annals of Behavior Medicine, Oxford, v. 40, n. 2, p. 184-19o, 2010. DOI: 10.1007/s12160-010-9200-X

CENSIDA - CENTRO NACIONAL PARA LA PREVENCIÓN Y EL CONTROL DEL VIH/SIDA. Guía de manejo antirretroviral de las personas con VIH. 5. ed. Ciudad de México: 2012.

CENSIDA - CENTRO NACIONAL PARA LA PREVENCIÓN Y EL CONTROL DEL VIH/SIDA. Informe nacional del monitoreo de compromisos y objetivos ampliados para poner fin al sida. Ciudad de México: Secretaría de Salud, 2018.

CENSIDA - CENTRO NACIONAL PARA LA PREVENCIÓN Y EL CONTROL DEL VIH/SIDA. Día mundial del SIDA. Ciudad de México: Secretaría de Salud, 2019a.

COMPEÁN, S.; PÉREZ-SALGADO, D.; STAINESOROZCO, M. G. et al. Satisfaction with healthcare services and adherence to antiretroviral therapy among patients with HIV attending two public institutions. Revista de Investigación, Mexico City, v. 67, n. 2, p. 8o-88, 2015.

CURRAN, K.; BAETEN, J.; COATES, T. et al. HIV-1 prevention for HIV-1 serodiscordant couples. Current HIV/AIDS Reports, Berlin, v. 9, n. 2, p. 16o-170, 2012. DOI: 10.1007/s11904-012-0114-Z DOAN VAN, E.; MEREISH, E.; WOULFE, J. et al. Perceived discrimination, coping mechanisms, and effects on health in bisexual y other nonmonosexual adults. Archives of Sexual Behavior,
Berlin, v. 48, n. 1, p. 159-174, 2019. DOI: 10.1007/ s10508-018-1254-Z

GALVAN, F.; BOGART, L.; KLEIN, D. et al. Medical mistrust as a key mediator in the association between perceived discrimination y adherence to antiretroviral therapy among HIV-positive Latino men. Journal of Behavioral Medicine, Berlin, v. 40, n. 5, p. 784-793, 2017. DOI: 10.1007/s10865-017-9843-1 GAMAREL, K.; NEILANDS, T.; DILWORTH, S. et al. Smoking, internalized heterosexism, y HIV disease management among male couples. AIDS Care, Abingdon-on-Thames, v. 27, n. 5, p. 649-654, 2015. DOI: 10.1080/09540121.2014.991269

GARCÍA, J.; PARKER, C.; PARKER, R. et al. Psychosocial implications of homophobia and HIV stigma in social support networks: insights for high-impact HIV prevention among black men who have sex with Men. Health Education \& Behavior, Thousand Oaks, v. 43, n. 2, p. 217-225, 2016. DOI: 10.1177/1090198115599398

GOBIERNO DE MÉXICO. ¿Qué es la Profilaxis Pre Exposición (PrEP)?, 2019b. Disponível em: $<$ https://bit.ly/2TmLcAI>. Acesso em: 15 set. 2020.

HATZENBUEHLER, M.; PACHANKIS, J. Stigma y minority stress as social determinants of health among lesbian, gay, bisexual, y transgender youth: research evidence y clinical implications. Pediatric Clinics of North America, Amsterdam, v. 63, n. 6, p. 985-997, 2016. DOI: 10.1016/j.pcl.2016.07.003

JOHNSON, M.; CARRICO, A.; CHESNEY, M. et al. Internalized heterosexism among HIVpositive, gay-identified men: implications for HIV prevention y care. Journal of Consult Clinical Psychology, Washington, DC, v. 76, n. 5, p. 829-839, 2008. DOI: 10.1037/0022-006X.76.5.829

MCGARRITY, L. Socioeconomic status as context for minority stress y health disparities among lesbian, gay, y bisexual Individuals. Psychology of Sexual Orientation y Gender Diversity, Washington, DC, v. 1, n. 4, p. 383-397, 2014. DOI: $10.1037 /$ sgdooooo67

MENDOZA-PÉREZ, J.; ORTIZ-HERNÁNDEZ, L. Association between overt and subtle experiences of discrimination and violence and 
mental health in homosexual and bisexual men in Mexico. Journal of Interpersonal Violence, Thousand Oaks, 886260519898423, 2020. DOI: $10.1177 / 0886260519898423$

MEYER, I. Prejudice, social stress, y mental health in lesbian, gay, y bisexual populations: conceptual issues y research evidence. Psychology Bulletin, Washington, DC, v. 29, n. 5, p. 674-697, 2003. DOI: 10.1037/0033-2909.129.5.674

NAKAGAWA, F.; MAY, M.; PHILLIPS, A. Life expectancy living with HIV: recent estimates y future implications. Current Opinion in Infectious Diseases, Philadelphia, v. 26, n. 1, p. 17-25, 2013. DOI: 10.1097/QCO.obo13e32835ba6b1

NIEUWKERK, P.; OORT, F. Self-reported adherence to antiretroviral therapy for HIV-1 infection y virologic treatment response: a metaanalysis. Journal of Acquired Immune Deficiencies Syndroms, Philadelphia, v. 38, n. 4, p. 445-448, 2005. DOI: 10.1097/o1.qai.oooo147522.34369.12 OMS - ORGANIZACIÓN MUNDIAL DE LA SALUD. Adherencia a los tratamientos a largo plazo: pruebas para la acción. Génova: 2004.

OMS - ORGANIZACIÓN MUNDIAL DE LA SALUD. VIH/SIDA, 2019. Disponível em: <https://bit. ly/2Tn4EoA>. Acesso em: 15 set. 2020.

ORTIZ-HERNÁNDEZ, L. La opresión de minorías sexuales desde la inequidad de género. Política y Cultura, Ciudad de México, n. 22, p. 161-182, 2004.

ORTIZ-HERNÁNDEZ, L. Influencia de la opresión internalizada sobre la salud mental de bisexuales, lesbianas y homosexuales de la Ciudad de México. Salud Mental, Ciudad de México, v. 28, n. 4, p. 49-65, 2005.

ORTIZ-HERNANDEZ, L.; GARCÍA, M. Opresión internalizada y prácticas sexuales de riesgo en varones homo-y bi-sexuales de México. Revista de Saúde Pública, São Paulo, v. 39, n. 6, p. 956-964, 2005a. DOI: 10.1590/So034-89102005000600014 ORTIZ-HERNÁNDEZ, L.; GARCÍA, M. Efectos de la violencia y la discriminación en la salud mental de bisexuales, lesbianas y homosexuales de la Ciudad de México. Cadernos de Saúde
Pública, São Paulo, v. 21, n. 3, p. 913-925, 2005b. DOI: 10.1590/So102-311X2005000300026

ORTIZ-HERNÁNDEZ, L.; PÉREZ-SALGADO, D.; COMPEAN-DARDÓN, S. Inseguridad alimentaria y adherencia al tratamiento antirretroviral en personas con VIH de México. Ciência \& Saúde Coletiva, Rio de Janeiro, v. 22, n. 2, p. 543-551, 2016. DOI: $10.1590 / 1413-81232017222.10792016$

REMOR, E. Fiabilidad y validez de la versión española del cuestionario MOS-SF-3o para evaluar la calidad de vida de personas infectadas por el VIH. Atención primaria, España, v.32, n.1, p. 1-12, 2003. DOI: 10.1016/So212-6567(03)78849-0

MÉXICO. Informe epidemiológico del VIH/SIDA en la Ciudad de México, 2013. Ciudad de México: Programa de VIH/SIDA de la Ciudad de México, 2013.

SHERBOURNE, C. D.; STEWART, A. L. The MOS social support survey. Social Science \& Medicine, Amsterdam, v. 32, n. 6, p. 705-714, 1991. DOI: 10.1016/0277-9536(91)90150-B

UNAIDS. Fact sheet 2021. Geneva: 2020.

\section{Contribución de los autores}

Ortiz-Hernández, contribuyó en el diseño de la investigación, el procesamiento de la información. Pérez-Salgado y MirandaQuezada, contribuyeron en,el procesamiento de la información. Staines-Orozco y Compean Dardón contribuyeron en el diseño de la investigación. Todos contribuyeron en la discusión de resultados, así como en la elaboración del artículo.

Recibido: 29/04/2021

Re-presentado: 06/10/2020; 11/12/2020; 08/03/2021; 29/04/2021 Aprobado: 10/05/2021 